\title{
Aproximación a un modelo de intervención psicológica en atención primaria basado en la terapia breve
}

\section{Approach to a model of psychological intervention in primary care based on brief therapy}

Fecha de recepción: 03--04-2018

Fecha de aceptación: 24-06-2018
Mario Gálvez-Lara*

Jorge Corpas*

Eliana Moreno*

José Fernando Venceslá*

Francisco García-Torres*

Juan Antonio Moriana*

* Departamento de Psicología de la Universidad de Córdoba Instituto Maimónides para la Investigación Biomédica (Córdoba)

Hospital Reina Sofía (Córdoba)

\section{resumen/ahstract:}

El incremento de consultas relacionadas con problemas y trastornos mentales en Atención Primaria y las características propias de este nivel asistencial (masificación de pacientes, hiperfrecuentación, etc.), hacen que cobre sentido el debate sobre la participación de los profesionales de la psicología en este contexto. Además, en los últimos años se ha reivindicado una mejor adecuación de los tratamientos psicológicos a las situaciones reales de aplicación, lo que conllevaría, entre otras actuaciones, su adaptación a formatos breves de tiempo limitado. En el presente trabajo analizamos la importancia que tiene la psicología en los problemas de salud dentro del sistema público de salud, incidiendo en las principales problemáticas y en el estado actual de la situación en nuestro país, así como en el surgimiento de las terapias breves como una opción terapéutica de bajo coste para responder a las exigencias de los sistemas públicos de salud acerca del uso de terapias psicológicas de corta duración que generen resultados favorables en la práctica clínica.

The increase of consultations related to problems and mental disorders in Primary Care and the characteristics of this level of care (mass of patients, hyper-attendance, etc.), add meaning to the debate on the participation of psychologists in this context. Furthermore, in recent years a better adaptation of psychological treatments to real application contexts has been claimed, what would entail, among other actions, its adjustment to brief formats of limited time. In the present work we analyze the importance of psychology in health problems within the public health system, focusing on the main issues and the current state of the situation in our country. At the same time, we reflect on the emergence of brief therapies as a low-cost therapeutic option to respond to the demands of the public health systems and how they are able to generate favorable results in clinical practice.

\section{palabras clave/keywords:}

Atención Primaria. Trastornos emocionales. Terapias breves.

Primary care. Emotional disorders. Brief therapies. 


\section{Introducción}

Sobre los años 80 se empezó a vislumbrar desde algunos foros la posibilidad de incluir a la psicología en los primeros escalones de los sistemas de salud para mejorar la calidad de vida de las personas y favorecer los hábitos saludables (Diekstra y Jansen, 1988). En la actualidad, la psicología clínica ya aparece como recurso de salud pública en los servicios de atención primaria (AP) de algunos países europeos. En España, a día de hoy, se están estudiando las posibilidades de la implementación de las terapias psicológicas en el contexto de AP. Concretamente, desde el año 2013 se está llevando a cabo un ensayo clínico a nivel nacional bajo el nombre de "Proyecto PsicAP", en el que se plantean tratamientos grupales para pacientes que sufran de ansiedad, estrés o depresión de gravedad leve o moderada. Concretamente, este proyecto pretende determinar si el tratamiento transdiagnóstico cognitivo-conductual, en formato grupal, es más efectivo y eficiente que el tratamiento habitual para los trastornos emocionales en los centros de AP españoles, así como comparar el efecto de ambos tratamientos en funcionalidad, calidad de vida, factores cognitivo-emocionales y satisfacción con el tratamiento (Cano-Vindel et al., 2016).

En la mayoría de los países europeos, el tratamiento que reciben las personas con trastornos emocionales de gravedad leve o moderada es fundamentalmente farmacológico debido a su abordaje casi exclusivo en AP (Kovess-Masfety et al., 2007). En este sentido, centrándonos en la población española, vemos que el tratamiento que reciben con mayor frecuencia los pacientes que presentan este tipo de trastornos implica la prescripción de psicofármacos $(35,3 \%)$ frente a la terapia psicológica $(4,5 \%)$ y la terapia combinada $(29,4 \%)$ (Codony et al., 2007). Además, no solo existe una falta de intervención psicológica, sino que la duración de los tratamientos con ansiolíticos, hipnóticos y antidepresivos es prolongada, ya que más del $50 \%$ de las prescripciones de fármacos tienen una duración superior a un año (Vedia, Bonet, Forcada, y Parellada 2005). Esto provoca serios riesgos para la salud del paciente, debido a los efectos secundarios y al elevado índice de recaídas asociados a la medicación (Gill y Hatcher, 2000), y un coste muy elevado para la sanidad pública.

La tendencia generalizada al uso de fármacos para el tratamiento inicial de los problemas psicológicos contradice las indicaciones de las investigaciones y de las guías clínicas internacionales (NICE, 2011; Terluin et al., 2009; Van Marwijk et al., 2009), que aconsejan la utilización de terapias psicológicas como tratamiento de elección. Del mismo modo, se sugiere que, independientemente de la gravedad del caso, la terapia psicológica debería estar siempre presente (Gonçalves, González, y Vázquez, 2003; NICE, 2011). Otras investigaciones han demostrado que las terapias psicológicas son más eficaces que el tratamiento farmacológico para los trastornos ansioso-depresivos (Watts, Turnell, Kladnitski, Newby, y Andrews, 2015) y que los pacientes prefieren ser tratados con terapias psicológicas, ya que son más acordes a su concepción de la problemática que presentan (Prins, Verhaak, Bensing, y van der Meer, 2008; Walters, Buszewicz, Weich, y King, 2008). De este modo, parece incongruente que la ciencia apunte hacia una dirección para la mejora de la salud pública, y la práctica clínica no asuma lo que indican los resultados de la investigación. 
A pesar de la importancia de evaluar la eficacia de los tratamientos psicológicos (GálvezLara et al., 2018; Moriana, Gálvez-Lara, y Corpas, 2017), el conocimiento de los resultados de la investigación empírica y su integración posterior por parte del profesional en la práctica clínica no llega a consolidarse del todo en los ámbitos aplicados, teniendo poco impacto sobre la práctica del profesional (Kazdin, 2011). Esta situación ha promovido la reivindicación de una mejor adecuación de los tratamientos a las situaciones reales de aplicación (Kazdin, 2008), lo que conllevaría su flexibilización y utilización en diferentes contextos (atención primaria y especializada), su adaptación a formatos breves de tiempo limitado y la generalización de las terapias a otros "problemas" psicológicos (no necesariamente trastornos) o circunstancias psicosociales en las que pueda cobrar sentido la participación de psicólogos o psicólogas (Moriana y Martínez, 2011).

En los últimos años se está sugiriendo la posibilidad de adaptar las terapias psicológicas tradicionales a un formato breve de tiempo limitado como posible solución realista para el tratamiento correcto de los trastornos mentales comunes (Shepardson, Funderburk y Weisberg, 2016), ajustándose más a la dinámica real de los servicios de salud y a las demandas de los usuarios. Algunos estudios han demostrado que cuando se incluía a profesionales de la psicología clínica con experiencia en terapias breves en el contexto de AP para el tratamiento de los trastornos ansioso-depresivos, se apreciaban beneficios sustanciales tanto para los pacientes como para el ejercicio profesional de los facultativos (Dath, Dong, Stewart, y Sables, 2014). En este sentido, invertir en tratamientos psicológicos breves para atender los problemas comunes en AP y atención especializada (AE) mejoraría la sintomatología de los pacientes, reduciría la hiperfrecuentación, y disminuiría la prescripción de fármacos y la presión asistencial que suponen este tipo de trastornos (Cape, Whittington, Buszewicz, Wallace, y Underwood, 2010). Por estos motivos, algunos autores recomiendan su inserción en la red pública de salud, apuntando que quizá puedan ser la solución para la descongestión y mejor funcionamiento de todo el sistema (Cape et al., 2010; Churchill et al, 2001).

\section{Estado de la cuestión}

\section{Conceptualización de las terapias breves}

La "terapia breve" o "terapia de tiempo limitado" surge como una opción terapéutica de bajo coste para responder a las exigencias de los sistemas públicos de salud acerca del uso de terapias psicológicas de corta duración que generen resultados favorables en la práctica clínica (Hewitt y Gantiva, 2009). Este tipo de terapias es utilizado por numerosos modelos teóricos que reducen su método tradicional de tratamiento a formatos de tiempo limitado (Epstein y Brown, 2002), entre los que se encuentran la terapia psicodinámica breve, la terapia interaccional estratégica breve, la terapia breve cognitivo-conductual, la psicoterapia expresiva de apoyo, la terapia de solución de problemas, la terapia centrada en la realización de tareas, la intervención en crisis, la terapia breve de familia o la terapia breve de grupo.

Siguiendo a Cape et al. (2010), para que una terapia se considere breve debe tener más de dos sesiones y menos de diez, estableciendo una media de seis sesiones, partiendo de la idea de que las guías son flexibles a las características y síntomas del paciente. Si tenemos en cuenta que la mayoría de las personas interrumpen la psicoterapia cuando únicamente 
han realizado unas pocas sesiones, cobra sentido organizar la terapia en torno al número de sesiones a las que acude el grueso de los pacientes. Aunque no existe un acuerdo entre diferentes autores acerca de cuantas sesiones comprenden las terapias de tiempo limitado, todos coinciden en la importancia del tiempo como herramienta terapéutica (Bedics, Henry, y Atkins, 2005; Hewitt y Gantiva, 2009; Lyons y Low, 2009; MacNeil, 2001; Miller, 2000). La limitación del número de sesiones ayuda a que tanto terapeuta como paciente se centren por completo en la terapia, aumenta la motivación del paciente y requiere que el terapeuta sea activo en el establecimiento de metas alcanzables, planteando cada sesión como una intervención con un resultado particular con el objetivo de que el paciente experimente el cambio lo antes posible (Fosha, 2004).

En conflicto con la idea de que la terapia psicológica requiere mucho tiempo y es costosa, la terapia breve se ha desarrollado para responder de manera efectiva y ética a los recursos económicos y las necesidades psicológicas de las personas (Lyons y Low, 2009). Debido a su duración y bajo coste, muchos usuarios de los servicios públicos de salud podrían beneficiarse. Además, la terapia breve podría utilizarse para ofrecer terapia psicológica inmediata a aquellos pacientes que están en listas de espera para acceder a programas especializados, como tratamiento inicial para usuarios de riesgo y como un complemento a tratamientos psicológicos más extensos (Sánchez y Gradolí, 2002). Debido a su idiosincrasia, estas terapias están especialmente indicadas para los problemas adaptativos y problemas emocionales de gravedad leve o moderada y se sugiere que deberían ser el primer paso para el abordaje terapéutico en este perfil de pacientes, dando una amplia accesibilidad a los pacientes y una respuesta eficaz a sus dolencias (Collings et al., 2015).

\section{La efectividad de las terapias breves}

Las terapias breves permiten que la mayoría de los pacientes con problemas emocionales con síntomas leves-moderados puedan ser ayudados con relativa rapidez, ya que algunas investigaciones sobre la eficacia de estas terapias han mostrado que obtienen resultados similares a las terapias convencionales y que, en la mayor parte de los casos, las principales mejorías en el paciente ocurren dentro de las diez primeras sesiones (Bloom, 2001; Lyons y Low, 2009; Miller, 2000). Por tanto, este tipo de terapias se equiparan a las tradicionales en términos de eficacia, siendo mejores en términos de eficiencia y de aceptación por parte de los pacientes (Churchill et al., 2001; Nieuwsma, Trivedi, McDuffie, Kronish, Benjamin, y Williams, 2012).

Un estudio meta-analítico con 34 estudios realizado por Cape et al. (2010) señala la efectividad de varias terapias en formato de tiempo limitado (terapia cognitivo conductual, counselling y terapia de solución de problemas) para problemas comunes en contextos de AP, recomendando la inserción de tratamientos psicológicos breves en dicho nivel de atención y señalando la necesidad de ampliar el nivel de evidencia y depurar los procedimientos para el diseño e implementación de este tipo de intervenciones. Estos autores destacan, además, que la efectividad de la terapia breve cognitivo conductual para los trastornos de ansiedad es similar a la del tratamiento extenso. 
Las terapias breves han demostrado su efectividad en la reducción de síntomas ansiosodepresivos (Amaya, Cardona, Ramírez, Sánchez, y Gantiva, 2008; Arco, López, Heilborn, y Fernández, 2005; Bernhardsdottir, Vilhjalmsson, y Champion, 2013; Koutra, Katsiadrami, y Diakogiannis 2010, Saravanan, Alias, y Mohamad, 2017), en la mejora de las habilidades de solución de problemas (Bannink, 2007), en la disminución de síntomas de estrés postraumático (Kip et al., 2016; Labrador, Fernández, y Rincón, 2006), o en el descenso de la ingesta de alcohol en personas que presentaban un consumo excesivo (Gantiva, Gómez, y Flórez, 2003). Además, las terapias breves no sólo han demostrado su efectividad inmediatamente después del tratamiento, sino que la mejoría en el paciente permanece tiempo después de finalizar la intervención (Hamdan-Mansour, Puskar, y Bandak, 2009; Vázquez et al., 2012). Así mismo, desde una perspectiva transdiagnóstica enfocada a las terapias breves para el abordaje de los trastornos emocionales, se recogen varios estudios que avalan su eficacia tanto para la reducción de la sintomatología como para la aplicación del tratamiento a un amplio número de pacientes con características distintas y trastornos comórbidos (Barlow, Allen y Choate, 2004; Brown y Barlow, 2009; McManus, Shafran, y Cooper, 2011; Norton, 2008).

Sin embargo, a pesar de los beneficios que han demostrado este tipo de terapias, algunos trabajos dudan sobre su eficacia real (Hemmings, 2000), cuestionando el mantenimiento de los resultados a largo plazo, el diseño de las intervenciones, la ambigüedad de la metodología aplicada y las dificultades que presentan para incluirse como un recurso accesible para toda la ciudadanía (Seekles et al., 2013).

\section{Consideraciones para aplicar la terapia y el consejo psicológico breve en AP}

Buena parte de las consultas susceptibles de intervención psicológica en AP no suelen ser trastornos psicológicos graves y muchos de ellos son problemas muy relacionados con cuestiones de la vida cotidiana y con síntomas subumbral. En este sentido, a la hora de intervenir con pacientes que presenten este tipo de problemáticas sería recomendable tener en cuenta las consideraciones que reflejamos a continuación:

1. El punto de partida de las terapias breves. Resulta fundamental establecer desde el primer momento la contextualización en la que se dan este tipo de terapias y hacer consciente al usuario de que se va a implementar una intervención de tiempo limitado. El objetivo no es tratar temas biográficos de alta repercusión con un modelo psicoterapéutico de largo recorrido que trate los problemas de fondo, complejos y del itinerario vital del individuo. Este tipo de terapias van más indicadas a problemas específicos, situacionales, que han generado un efecto negativo en la persona. Se intenta predeterminar un intervalo aproximado de sesiones tras la evaluación inicial para pactar los objetivos específicos de la intervención para dicho intervalo de tiempo. También se subraya la importancia de la motivación al cambio y de la activación de los recursos del individuo mucho más que en el entrenamiento de nuevas habilidades que pudieran requerir de más tiempo.

2. Psicoeducación. Cuando a los pacientes se les explica la etiología o fuente de su conflicto, sus posibles cursos y evoluciones y se le dan alternativas fáciles para su manejo, mejoramos la competencia de muchos de ellos y la comprensión de lo que les ocurre. Sabemos 
de la importancia que la información tiene para la comprensión de la mayoría de problemas de salud y como ella proporciona a veces motores de cambio y afrontamiento, sobre todo de problemas menores.

3. Las técnicas de resolución de problemas. Muchos de los problemas cotidianos de los usuarios de AP con síntomas ansioso-depresivos están asociados a la dificultad para resolver problemas cotidianos. La aplicación de técnicas de resolución de problemas por profesionales de la psicología con experiencia en terapias breves puede suponer un importante empuje a la raíz del origen de muchos síntomas que pueden facilitar una rápida resolución o atenuación de conflictos.

4. El afrontamiento. El uso de técnicas de afrontamiento que ayuden a los pacientes a hacer frente a las demandas internas y del ambiente que les puedan resultar estresantes o ansiógenas es fundamental para la reducción de sus síntomas. Desde muchos enfoques, tanto clásicos como modernos, hemos aprendido técnicas de afrontamiento para múltiples situaciones relacionadas tanto con enfermedades crónicas, dolor, etc., como con situaciones psicosociales habituales como la pérdida y el duelo. Existen incluso modelos específicos según estas circunstancias que pueden facilitar al profesional de la psicología en AP paliar y orientar el afrontamiento a una dirección preventiva que frene el inicio de un problema mayor.

5. La psicopatologización de la vida cotidiana. En la actualidad, cuestiones de la vida cotidiana, como que un niño se orine en la cama con tres años o la muerte de un familiar, se convierten en hechos diagnosticables y psicopatologizantes a nivel individual y familiar. Trabajar en AP desde un enfoque normalizador, no psicopatológico, para multitud de problemas, puede normalizar la vida de un individuo de una forma mucho más saludable que recomendando fármacos que solo deberían prescribirse para los casos más graves.

6. Enfoques centrados en el problema y en el aquí y en el ahora. En el contexto de las terapias breves, focalizar la atención del paciente en el problema específico que genera su malestar ( si es solo un problema) y en el momento actual es mucho mejor que centrarnos en aspectos históricos y biográficos normalmente asociados a mayores y peores problemas. Trabajar en lograr un posicionamiento del paciente en un paradigma centrado en estos aspectos es muy importante para las terapias breves y para la búsqueda del cambio.

7. Las fortalezas. En línea totalmente opuesta a la anterior, un planteamiento centrado en las fortalezas es más constructivo y genera mejores resultados que uno centrado en las debilidades, sobre todo en los casos más leves.

8. El cambio de esquemas. Muchos modelos se han centrado en esquemas o en profundos cambios o reestructuraciones de pensamiento. Es cierto que cambios de gran calado han estado asociados a modelos psicoterapéuticos de largo recorrido y extensas terapias dirigidas a los cimientos de las estructuras fundamentales de los individuos, pero en el contexto de la terapia breve es difícil situarse en este plano con importantes limitaciones de tiempo y sesiones. Sin embargo, existen modelos que propugnan el trabajar el cambio de esquemas y reestructuración o modificación de pensamientos desde una perspectiva centrada en una idea, creencia o problema que tenga una relevancia de interés en un caso en concreto. 
9. La motivación. Probablemente, la motivación por la vida, la motivación al cambio y la motivación para superar las dificultades son los motores más relevantes para potenciar dentro de un contexto de terapias breves. Activar un arsenal de motivación y compromiso con uno mismo es una de las herramientas más importantes que puede utilizar un psicólogo o psicóloga, así como hacer responsable a los pacientes de sus propios avances y cambios. La motivación aumenta la capacidad de empoderamiento y la resiliencia.

10. Actividades complementarias. Aunque este tema, en ocasiones, no está suficientemente abordado desde la psicología, creemos que en el ámbito de la AP trabajar y orientar en cuestiones relacionadas con la planificación y organización de aspectos de la vida cotidiana (horarios, actividades, prioridades, elecciones cotidianas, actividades de ocio y tiempo libre, etc.), tienen, en muchas ocasiones, efectos muy relevantes en el cambio comportamental de las personas y en la activación de las personas para realizar actividades tanto incompatibles con algunos padecimientos como motivadoras y reforzantes para complementar los efectos del resto de técnicas implementadas.

11. Técnicas específicas. Además de lo expuesto con anterioridad, una vez evaluado el problema inicial habrá además de algunas cuestiones de posicionamiento inicial, técnicas específicas para problemas específicos que justificarán su elección (por ejemplo, trabajar de forma concreta para aumentar la autoestima, reestructuración de pensamientos, técnicas de relajación o mindfulness, etc.)

12. Consideraciones para la atención infantojuvenil. Uno de los temas menos tratados en la literatura es la atención psicológica en el contexto de la AP en niños y adolescentes. Aunque algunos países tienen unidades específicas de atención infantojuvenil, dichas unidades (además de saturadas) suelen estar enfocadas a problemas graves de comportamiento y del desarrollo. Sin embargo, existen una gran cantidad de problemas de índole leve y moderada, relacionados con el desarrollo evolutivo además de con problemas psicológicos, así como con una falta de información de los padres o de habilidades parentales adecuadas que generan un nivel de problemática muy elevado y que también colapsa los servicios de AP. En este sentido, la Pediatría supone una nueva dimensión de problemas que podrían equipararse a los de los adultos. Así, reivindicar la figura del profesional de la psicología clínica infantil en el contexto de AP también tendría una gran repercusión, especialmente en muchos problemas en los que ni siquiera es necesario tratar al niño porque buena parte de este tipo de abordajes se efectúan solamente con los padres o cuidadores principales.

\section{Protocolo de terapia breve cognitivo-conductual}

Como hemos planteado anteriormente, las terapias breves son utilizadas por numerosos modelos teóricos que reducen su método tradicional de tratamiento a formatos de tiempo limitado. Dentro de la perspectiva cognitivo-conductual, la intervención breve se centra en animar al paciente a que contraste empíricamente sus ideas y creencias, identificando los antecedentes y reforzadores de la conducta desadaptativa con el objetivo de que éste desarrolle y potencie habilidades para afrontar y prevenir situaciones de riesgo de forma exitosa. Siguiendo a Hewitt y Gantiva (2009), este modelo de terapia breve se centra en el presente más que en la historia lejana del paciente, pretende incrementar la motivación y la autoefi- 
cacia hacia el cambio, trabaja los pensamientos y las emociones para lograr el cambio y tiene como objetivo la toma de decisiones y el inicio del cambio comportamental.

A continuación, presentaremos una propuesta de terapia breve cognitivo conductual basada en el protocolo unificado para el tratamiento transdiagnóstico de los trastornos emocionales (PU; Barlow et al., 2015). Este protocolo ha sido desarrollado para abordar los síntomas subyacentes a los trastornos de ansiedad, trastornos del estado de ánimo y otros trastornos relacionados en los que la ansiedad y la desregulación emocional ejercen un papel importante (Ellard, Fairholme, Boisseau, Farchione, y Barlow, 2010). El PU se puede aplicar simultáneamente a pacientes con variedad de trastornos y puede llevarse a cabo en formato grupal fácilmente, por lo que podría reducir las listas de espera y el coste del tratamiento individual (Osma et al., 2018).

La eficacia del PU ha sido demostrada tanto en formato individual (Farchione et al., 2012) como en formato grupal (Bullis et al., 2015). En España, el estudio preliminar de Osma et al. (2015) obtuvo como resultado que, tras la aplicación del PU en formato grupal en una unidad de salud mental pública, la mayoría de los participantes dejaron de cumplir los criterios diagnósticos por los cuáles habían sido incluidos en el estudio, manteniéndose los beneficios del tratamiento a los 12 meses. En la actualidad, en nuestro país se están llevando a cabo varios ensayos clínicos para determinar la eficacia de este protocolo en diferentes contextos (García-Escalera et al., 2017; Osma et al., 2018).

La adaptación de este protocolo a un formato de tiempo limitado está compuesta por ocho sesiones:

Sesión 1. Mejorar la motivación y el compromiso con el tratamiento. El objetivo de esta sesión es mejorar la motivación para el cambio por parte del paciente, analizando la ventajas e inconvenientes de cambiar y estableciendo objetivos vitales significativos. Para ello utilizaremos una serie de estrategias de motivación para el cambio (ampliar la discrepancia entre la situación actual del paciente y su situación por cambiar, no confrontar al paciente ni ponerse directamente a favor del cambio, reforzar las afirmaciones de cambio de conductas positiva, etc.), una hoja de balance decisional en la que el paciente indicará los beneficios y los costes de cambiar o no cambiar, y una hoja de registro de objetivos realistas, alcanzables, concretos y fijados en el tiempo para aumentar las creencias del paciente en su capacidad para alcanzar con éxito el cambio.

Sesión 2. Entender las emociones. Reconocimiento y análisis de la conciencia emocional. Esta sesión proporciona información sobre los roles y funciones adaptativas de las emociones usando ejemplos de la vida del paciente, introduce el concepto de conductas impulsadas por la emoción (CIEs), presenta el modelo de tres componentes de las emociones (pensamientos, sensaciones físicas y conductas) para que el paciente sea capaz de distinguir entre lo que pensamos, lo que sentimos y los que hacemos, e introduce el análisis de las experiencias emocionales (antecedentes, respuestas emocionales y consecuencias) con el objetivo de que éste comprenda de dónde, cuándo y porqué ocurren las emociones.

Sesión 3. Entrenamiento en conciencia emocional: aprendiendo a observar las experiencias. En esta sesión se introducirá la conciencia emocional centrada en el presente, expli- 
cando cómo las reacciones secundarias a las emociones tienden a implicar un juicio crítico y una evaluación, y que en muchas ocasiones esas reacciones se basan en asociaciones y memorias relacionadas con experiencias pasadas. Además, se realizará un breve ejercicio de mindfulness para practicar la conciencia emocional centrada en el presente, sin juzgar.

Sesión 4. Evaluación cognitiva. El objetivo principal de esta sesión es ayudar a los pacientes a desarrollar patrones de pensamiento más flexibles, animando al paciente a que vea lo que ve tal y como lo ve, sin juzgar ni justificar cualquier tipo de contenido. Por ejemplo, cambiar la expresión "Yo nunca me enfado con la gente" por "En este momento, me estoy sintiendo enfadado". Para ello podemos utilizar la técnica de la flecha descendente, autorregistros (estímulo desencadenante, respuesta emocional y pensamientos asociados), además de mostrarles cómo identificar formas de pensamiento inadaptadas y cómo modificarlas.

Sesión 5. Evitación emocional y CIEs. Los propósitos de esta sesión son enseñar a los pacientes que las estrategias de evitación emocional contribuyen al mantenimiento de los trastornos emocionales y ayudarlos a reemplazar sus propias CIEs inadaptadas por otros comportamientos más adaptativos. En primer lugar, se puede utilizar el experimento del "oso blanco" para demostrar el papel contraproducente que juega la evitación emocional en ciertas ocasiones. A continuación, una vez que el paciente haya aprendido a identificar los patrones de evitación emocional y las CIEs, se trabajará para intentar contrarrestar estas conductas mediante la exposición a las situaciones que desencadenan dichas emociones y la realización de conductas alternativas a las CIEs.

Sesión 6. Conciencia y tolerancia a las sensaciones físicas. Esta sesión se centra en el papel que las sensaciones físicas ejercen sobre la respuesta emocional y en el entrenamiento de los pacientes en exposición interoceptiva para promover la habituación a dichas sensaciones. Con este objetivo, explicaremos al paciente que la interpretación que hace de sus sensaciones físicas tiene influencia en la manera en que éste manifiesta y experimenta la respuesta emocional, y realizaremos una serie de ejercicios de inducción de síntomas que ayudarán a aumentar la conciencia de las sensaciones físicas como parte de la repuesta emocional, a aumentar la tolerancia a estas sensaciones y a reducir los patrones de evitación. Un ejemplo de estos ejercicios podría ser hiperventilar voluntariamente, respirar a través de una pajita, dar vueltas sobre uno mismo o correr sin desplazarse del sitio.

Sesión 7. Exposición emocional interoceptiva y situacional. El propósito de esta sesión es exponer al paciente a los desencadenantes internos y externos que le producen intensas reacciones emocionales, con el fin de aumentar la tolerancia a las emociones y reducir el comportamiento de evitación. Es útil utilizar jerarquías de evitación emocional y situacional para tener claro el tipo de situaciones que desencadenan emociones desagradables en el paciente y las situaciones que evita más a menudo. Uno de los elementos cruciales del éxito del tratamiento está en la práctica repetida de exposición a emociones fuera de la consulta, por lo que el terapeuta y el paciente deben trabajar juntos para diseñar las exposiciones entre sesiones. 
Sesión 8. Logros, mantenimiento y prevención de recaídas. En esta última sesión se pretende discutir las habilidades aprendidas durante el tratamiento mediante el uso de un registro de autoevaluación del progreso, identificar las habilidades que se deben seguir practicando en el futuro y enseñar a identificar y enfrentar situaciones futuras de alto riesgo.

\section{Conclusiones}

La realidad asistencial de los trastornos mentales comunes en el sistema público de salud es mejorable y demanda un cambio en los paradigmas de tratamiento. Los médicos de familia son los primeros en recibir a los pacientes con un posible trastorno o problema mental. Como ya hemos comentado, gran parte de los casos son tratados con psicofármacos como primera opción terapéutica por esos mismos profesionales en AP (Secades Villa et al., 2003). Sin embargo, también es cierto que se producen algunas derivaciones a salud mental especializada de pacientes sin un trastorno mental diagnosticable, principalmente con problemas interpersonales o síntomas ansioso-depresivos (Lobo, González, y Rodríguez, 2006), produciéndose un colapso de la atención especializada en salud metal.

En este contexto, hay sistemas públicos como el británico que, para este tipo de pacientes, recomienda la intervención psicológica durante al menos cinco semanas antes de recurrir a la terapia farmacológica. Además, Gran Bretaña, en el año 2008, puso en marcha el programa IAPT (Improving Access to Psychological Therapies) para atender en AP a los trastornos mentales comunes de gravedad leve/moderada con intervenciones de baja intensidad. Los resultados de diversos estudios para la evaluación de este programa han mostrado que más del 50\% de los pacientes se recuperaron con pocas sesiones y que los cambios eran duraderos en el tiempo (Clarck et al., 2009; Gyani, Shafran, Layard y Clark, 2013). Además, se calcula que el programa IAPT es más económico en términos de costes-beneficios en comparación con el tratamiento especializado de alta intensidad (Radhakrishnan et al., 2013). En España, quizá el "Proyecto PsicAP" sea el primer paso para la incorporación de psicólogos y psicólogas en AP, tal y como recomiendan la mayoría de médicos de familia (Moreno y Moriana, 2012).

Por lo tanto, las terapias breves podrían ser una posible solución para el abordaje de los problemas mentales en el sistema público de salud, al considerarse una opción tanto en los servicios de AP como en los de AE. Sin embargo, los datos siguen siendo escasos y no se han identificado aspectos relevantes respecto a las características de los pacientes o la forma adecuada de implementación, debido a lo cual se hacen necesarios más ensayos clínicos que arrojen luz sobre la idoneidad de estos abordajes.

\section{Referencias}

Amaya, M., Cardona, S., Ramírez, S., Sánchez, L., y Gantiva, C. (2008). Evaluación de la efectividad de un programa de intervención breve para la disminución de la ansiedad en personas que ingresan por primera vez a un centro penitenciario. Psychologia. Avances de la Disciplina, 2(1), 79-96. 
Arco, J., López, S., Heilborn, V., y Fernández, F. (2005). Terapia breve en estudiantes universitarios con problemas rendimiento académico y ansiedad: Eficacia del modelo "La Cartuja". International Journal of Clinical and Health Psychology, 5(3), 589-608.

Bannink, F. (2007). Solution-focused brief therapy. Contemporary Psychotherapy, 37, 87-94.

Barlow, D. H., Allen, L. B., y Choate, M. L. (2004). Toward a unified treatment for emotional disorders. Behavioral Therapy, 35, 205-230.

Barlow, D. H., Farchione, T. J., Fairholme, C. P., Ellard, K. K., Boisseau, C. L. Allen, L. B., y Ehrenreich-May, J. (2015). Protocolo Unificado para el tratamiento transdiagnóstico de los trastornos emocionales: manual del terapeuta y manual del paciente. Madrid: Alianza Editorial.

Bedics, J. D., Henry, W. P., y Atkins, D. C. (2005). The therapeutic process as a predictor of change in patients' important relationships during time-limited dynamic psychotherapy. Psychotherapy Theory, Research, Practice, Training, 42, 279-284.

Bernhardsdottir, J., Vilhjalmsson, R., y Champion, J. D. (2013) Evaluation of a brief cognitive behavioral group therapy for psychological distress among female Icelandic University students. Issues in Mental Health Nursing, 34, 497-504

Bloom, B. L. (2001). Planned short-term psychotherapy for depression: Recent controlled outcome studies. Brief Treatment and Crisis Intervention, 1, 169-189.

Brown, T. A. y Barlow, D. H. (2009). A proposal for a dimensional classification system based on the shared features of the DSM-IV anxiety and mood disorders: implications for assessment and treatment. Psychological Assessment, 21, 256-271.

Bullis, J. R., Sauer-Zavala, S., Bentley, K. H., Thompson-Hollands, J., Carl, J. R., Barlow, D. H. (2015). The unified protocol for transdiagnostic treatment of emotional disorders: preliminary exploration of effectiveness for group delivery. Behavior Modification, 39(2), 295-321.

Cano-Vindel, A., Munoz-Navarro, R., Wood, C. M., Limonero, J. T., Medrano, L. A., Ruiz-Rodriguez, P., ... y Santolaya, F. (2016). Transdiagnostic cognitive behavioraltherapy versus treatment as usual in adult patients with emotional disorders inthe primary care setting (PsicAP Study): Protocol for a randomized controlledtrial. JMIR Research Protocols, 5, e246.

Cape, J., Whittington, C., Buszewicz, M., Wallace, P., y Underwood, L. (2010). Brief psychological therapies for anxiety and depression in primary care: meta-analysis and meta-regression. BMC Medicine, 8, 8-38.

Churchill, R., Hunot, V., Corney, R., Knapp, M., McGuire, H., Tylee, A., y Wessely, S. (2001). A systematic review of controlled trials of the effectiveness and cost-effectiveness of brief psychological treatments for depression. Health Technology Assessment, 35, 1-173.

Clark, D. M., Layard, R., Smithies, R., Richards, D. A., Sucking, R., y Wright, B. (2009). Improving access to psychological therapy: Initial evaluation of two UK demonstration sites. Behaviour Research and Therapy, 47, 910-920.

Codony, M., Alonso, J., Almansa, J., Vilagut, G., Domingo, A., Pinto-Meza, A., Fernández, A., Usall, J., Dolz, M., y Haro, J. M. (2007). Utilización de los servicios de salud mental en la población general española. Resultados del estudio ESEMeD-España. Actas Españolas de Psiquiatría, 35(2), 21-28.

Collings, S., Mathieson, F., Dowell, A., Stanley, J., Hatcher, S., Goodyear-Smith, F., Lane, B. y Munsterman, A. (2015). Clinical effectiveness of an ultra-brief intervention for common mental health syndromes in primary care: study protocol for a cluster randomized controlled trial. Trials, 16, 260. doi: 10.1186/s13063-015-0778-y.

Dath, S., Dong, C. Y., Stewart, W. M., y Sables, E. (2014). A clinical psychologist in GP-Land: an evaluation of brief psychological interventions in primary care. The New Zealand Medical Journal, 127, 62-73.

Diekstra, R. F. y Jansen, M. A. (1988). Psychology's role in the new health care systems: The importance of psychological interventions in primary health care. Psychotherapy: Theory, Research, Practice, Training, 25(3), 344-351.

Ellard, K. K., Fairholme, C. P., Boisseau, C. L., Farchione, T. J., y Barlow, D. H. (2010). Unified protocol for the transdiagnostic treatment of emotional disorders: Protocol development and initial outcome data. Cognitive and Behavioral Practice, 17, 88-101. 
Epstein, L. y Brown, L. (2002). Brief treatment and a new look at the task-centered approach. Boston: Allyn and Bacon.

Farchione, T. J., Fairholme, C. P., Ellard, K. K., Boisseau, C. L., Thompson-Hollands, J., Carl, J. R., Gallagher, M. W., Barlow, D. H. (2012). Unified protocol for transdiagnostic treatment of emotional disorders: a randomized controlled trial. Behavior Therapy, 43(3), 666-678.

Fosha, D. (2004). Brief integrative therapy comes of age: A commentary. Journal of Psychotherapy, 14, 66 - 92.

Gálvez-Lara, M., Corpas, J., Moreno, E., Venceslá, J. F., Sánchez-Raya, A., y Moriana, J. A. (2018). Psychological treatments for mental disorders in children and adolescents: a review of the evidence of leading international organizations. Clinical Child and Family Psychology Review. doi.org/10.1007/s10567-018-0257-6

García-Escalera, J., Valiente, R. M., Chorot, P., Ehrenreich-May, J., Kennedy, S. M., y Sandín, B. (2017). The Spanish Version of the Unified Protocol for Transdiagnostic Treatment of Emotional Disorders in Adolescents (UP-A) Adapted as a School-Based Anxiety and Depression Prevention Program: Study Protocol for a Cluster Randomized Controlled Trial. JMIR Research Protocol, 6(8): e149.

Gantiva, C., Gómez, C., y Flórez, L. (2003). Evaluación del impacto de un programa de autoayuda dirigida: una alternativa para el tratamiento de personas que abusan del consumo de alcohol. Revista Colombiana de Psicología, $12,38-48$.

Gill, D. y Hatcher, S. (2000). WITHDRAWN: antidepressants for depression in medical illness. Cochrane Database Systematic Reviews, CD001312.

Gonçalves, E. F., González, R. V. M., y Vázquez, C. J. (2003). Guía de Buena Práctica Clínica en Depresión y Ansiedad. Madrid: Ministerio de Sanidad y Consumo.

Gyani, A., Shafran, R., Layard, R., y Clark, D. M. (2013). Enhancing recovery rates: Lessons from year one of IAPT. Behaviour Research and Therapy, 51,597-606.

Hamdan-Mansour, A. M., Puskar, K., y Bandak, A. G. (2009). Effectiveness of cognitive-behavioral therapy on depressive symptomatology, stress and coping strategies among Jordanian university students. Issues in Mental Health Nursing, 30(3), 188-196.

Hemmings, A. (2000). A systematic review of the effectiveness of brief psychological therapies in primary health care. Families, Systems y Health, 18, 279-313.

Hewitt, N. y Gantiva, C. A. (2009). La terapie breve: una alternativa de intervención psicológica efectiva. Avances en Psicología Latinoamericana, 27(1), 165-176.

Kazdin, A. (2008). Evidence-Based Treatment and Practice. New opportunities to bridge clinical research and practice, enhance the knowledge base, and improve patient care. American Psychologist, 63, 146-159.

Kazdin, A. (2011). Evidence-Based Treatment Research: Advences, limitations and next steps. American Psychologist, $66,685-698$

Kip, K. E., D’Aoust, R. F., Hernandez, D. F., Girling, S. A., Cuttino, B., Long, M. K., Rojas, P., Wittenberg, T., Abhayakumar, A., y Rosenzweig, L. (2016). Evaluation of brief treatment of symptoms of psychological trauma among veterans residing in a homeless shelter by use of Accelerated Resolution Therapy. Nursing Outlook, 64(5), 411-423.

Koutra, A., Katsiadrami, A., y Diakogiannis, G. (2010). The effect of group psychological counselling in Greek university students' anxiety, depression, and self-esteem. European Journal of Psychotherapy y Counselling, 12(2), 101-111.

Kovess-Masfety, V., Alonso, J., Brugha, T. S., Angermeyer, M. C., Haro, J. M., y Sevilla-Dedieu, C. (2007). Differences in lifetime use of services for mental health problems in six European countries. Psychiatric Services, 58 213-220.

Labrador, F., Fernández, M., y Rincón, P. (2006). Eficacia de un programa de intervención individual y breve para el trastorno de estrés postraumático en mujeres víctimas de violencia doméstica. International Journal of Clinical and Health Psychology, 6(3), 527-547.

Lobo, 0. A., González, G. R., y Rodríguez, S. F. (2006). La derivación a salud mental de pacientes sin un trastorno psíquico diagnosticable. Atención Primaria, 38(10), 563-569. 
Lyons, R. y Low, P. (2009). Brief psychological therapy in primary care: The psychologist's challenge. New Zealand Journal of Psychology, 38(1), 24-31.

McManus, F., Shafran, R., y Cooper, Z. (2011). What does a transdiagnostic approach have to offer the treatment of anxiety disorders? British Journal of Clinical Psychology, 49, 491-505.

MacNeil, G. (2001). Time-limited psychosocial treatment for specific panic disorders and agoraphobia. Brief Treatment and Crisis Intervention, 1,29-41.

Miller, W. R. (2000). Rediscovering fire: Small interventions, large effects. Psychology of Addictive Behaviors, 14, 6-18.

Moriana, J. A., Gálvez-Lara, M., y Corpas, J. (2017). Psychological treatments for mental disorders in adults: A review of the evidence of leading international organizations. Clinical Psychology Review, 54, 29-43.

Moriana, J. A., y Martínez, V. (2011). La psicología basada en la evidencia y el diseño y evaluación de tratamientos psicológicos eficaces. Revista de Psicopatología y Psicología Clínica, 16, 81-100.

Moreno, E. M. y Moriana, J. A. (2012). El tratamiento de problemas psicológicos y de salud mental en atención primaria. Salud Mental, 35(4), 315-328.

National Institute for Health and Clinical Excellence (NICE). (2011). Common mental health problems: identification and pathways to care Clinical guideline. London: The British Psychological Society and The Royal College of Psychiatrists.

Nieuwsma, J. A., Trivedi, R. B., McDuffie, J., Kronish, I., Benjamin, D. y Williams, J. W. (2012). Brief psychotherapy for depression: a systematic review and meta-analysis. International Journal of Psychiatry Medicine, 43(2), 129-151.

Norton, P. J. (2008). An open trial of a transdiagnostic cognitive-behavioral group therapy for anxiety disorder. Behavior Therapy, 39, 242-250.

Osma, J., Suso-Ribera, C., García-Palacios, A., Crespo-Delgado, E., Robert-Flor, C., Sánchez-Guerrero, A., Ferreres-Galán, V., Pérez-Ayerra, L., Malea-Fernández, A., y Torres-Alfosea, M. A. (2018). Efficacy of the unified protocol for the treatment of emotional disorders in the Spanish public mental health system using a group format: study protocol for a multicenter, randomized, non-inferiority controlled trial. Health and Quality of Life Outcomes. doi.org/10.1186/s12955-018-0866-2

Prins, M. A., Verhaak, P. F. M, Bensing, J. M., y van der Meer, K. (2008). Health beliefs and perceived need for mental health care of anxiety and depression: the patients's perspective explored. Clinical Psychology Review, 28, 1038-1058.

Radhakrishnan, M., Hammond, G., Jones, P. B., Watson, A., McMillon-Shields, F., y Lafortune, L. (2013). Cost of Improving Access to Psychological Therapies (IAPT) programme: An analysis of cost of sessions, treatment and recovery in selected Primary Care Trusts in the East of England region. Behaviour Research and Therapy, 51, $37-45$.

Ruiz, A. (2004). La presión asistencial. Atención Primaria, 33(3), 145-148.

Sánchez, E. y Gradolí, V. (2002). Terapia breve en la adicción a drogas. Papeles del Psicólogo, 83, 49-54.

Saravanan, C., Alias, A., y Mohamad, M. (2017). The effects of brief individual cognitive behavioural therapy for depression and homesickness among international students in Malaysia. Journal of Affective Disorders, 220, 108-116.

Secades Villa, R., Rodríguez García, E., Valderrey Barbero, J., Fernández Hermida, J. R., Vallejo Seco, G., y Jiménez García, J. M. (2003). El consumo de psic ofármacos en pacientes que acuden a Atención Primaria en el Principado de Asturias (España). Psicothema, 15(4), 650-655.

Seekles, W., Cuijpers, P., Kok, R., Beekman, A., van Marwijk, H. y van Straten, A. (2013). Psychological treatment of anxiety in primary care: a meta-analysis. Psychological Medicine, 43, 351-361.

Shepardson, R. L., Funderburk, J. S. y Weisberg, R. B. (2016). Adapting evidence-based, cognitive-behavioral interventions for anxiety for use with adults in integrated primary care settings. Family, systems and health: the journal of collaborative family healthcare, 34(2), 114-127. 
Terluin, B., Van Heest, F. B., Van der Meer, K., Neomagus, G. J. H., Hekman, J., Aulbers, L. P. J., ... Grol, M. H. (2009). NHG Richtlijn Angststoornissen, M62, (herziening). [Guidelines anxiety disorders for general practice (revised)].

Van Marwijk, H. W. J., Grundmeijer, H. G. L. M., Bijl, D., Van Gelderen, M. G., De Haan M, Van Weel-Baumgarten, ... Romeijnders, A. C. M. (2009). NHG Richtlijn Depressieve stoornis, M44, (herziening). [Guidelines depressive disorders for general practice (revised)].

Vázquez, F. L., Torres, A., Blanco, V., Díaz, 0., Otero, P., y Hermida, E. (2012). Comparison of relaxation training with a cognitive-behavioural intervention for indicated prevention of depression in university students: A randomized controlled trial. Journal of Psychiatric Research, 46(11), 1456-1463.

Vedia, U. C., Bonet, M. S., Forcada, V. C., y Parellada, E. N. (2005). Estudio de utilización de psicofármacos en atención primaria. Atención Primaria, 36(5), 239-247.

Walters, K., Buszewicz, M., Weich, S., y King, M. (2008). Help-seeking preferences for psychological distress in primary care: effect of current mental state. British Journal of General Practice, 58, 694-698.

Watts, S. E., Turnell, A., Kladnitski, N., Newby, J. M., y Andrews, G. (2015). Treatment-as-usual (TAU) is anything but usual: a meta-analysis of CBT versus TAU for anxiety and depression. Journal of Affective Disorders, 175, $152-167$. 